\title{
High-Throughput and Label-Free Blood-on-a-Chip for Malaria Diagnosis
}

\author{
Yang Jun Kang, ${ }^{\dagger}$ Young-Ran Ha, ${ }^{\ddagger}$ and Sang-Joon Lee*,§ \\ ${ }^{\dagger}$ Department of Mechanical Engineering, Chosun University, Gwangju 501-759, Republic of Korea \\ ${ }^{\ddagger}$ Division of Integrative Biosciences and Biotechnology, Pohang University of Science and Technology (POSTECH), Pohang \\ 790-784, Republic of Korea \\ ${ }^{\S}$ Department of Mechanical Engineering, Pohang University of Science and Technology (POSTECH), Pohang 790-784, Republic of \\ Korea
}

Supporting Information

ABSTRACT: The malaria parasite Plasmodium falciparum (Pf) changes the structure and mechanical properties of red blood cells (RBCs). These changes decrease deformability and increase cytoadherence of Pf-infected RBCs to the vascular endothelium, eventually leading to flow occlusions in capillary vessels. In this study, to detect Pf-infected RBCs effectively, deformability and viscosity of blood sample are measured simultaneously and indirectly by quantifying blood flow in a microfluidic device. The microfluidic device is designed by mimicking a Wheatstone-bridge electric circuit. To measure RBC deformability, a deformability assessment chamber (DAC) at the left lower side channel has parallel microfluidic filters. After delivering blood sample and $1 \times$ PBS solution at the same flow rate, hemodynamic properties are
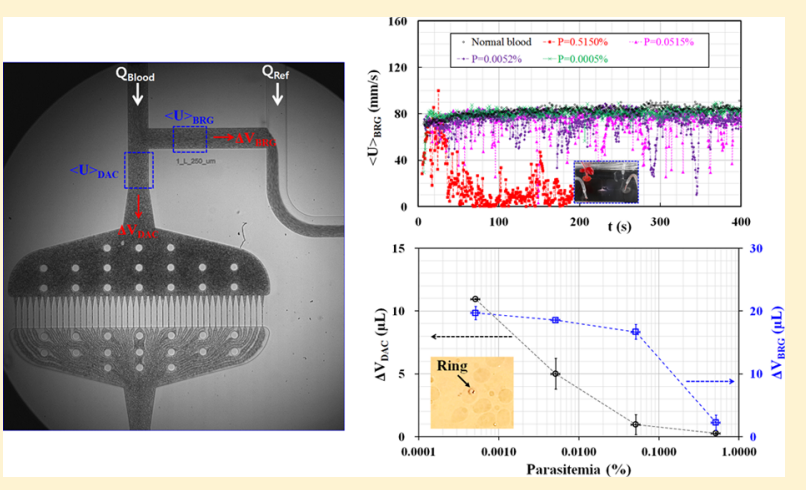
measured using a time-resolved microparticle image velocimetry technique. Blood volume delivered into the DAC for $200 \mathrm{~s}$ is evaluated as a deformability index. Subsequently, blood viscosity is quantified by monitoring blood-filled width of parallel flows in the microfluidic device. The proposed method is applied to evaluate variations in biophysical properties of blood samples partially mixed with normal RBCs and hardened RBCs. As a result, RBC deformability is more effective than blood viscosity in the detection of blood samples with hardened RBC volume fraction of $5 \%$. The microfluidic device is also applied to detect Pfinfected RBCs. When parasitemia is greater than $0.515 \%$ for ring stage, $0.0544 \%$ for trophozoite stage, and $0.0054 \%$ for schizont stage, the measured velocity fields show unstable behavior because of cytoadherence of Pf-infected RBCs. Blood volume delivered into the DAC significantly decreases with increasing parasitemia. The experimental method proposed in this study can detect Pf-infected RBCs with good accuracy.

A

mong various biophysical properties of blood, red blood cell (RBC) deformability has been considered a potential biomarker for monitoring pathological changes related to various hematologic diseases. ${ }^{1}$ RBC deformability depends on several factors, including membrane cytoskeleton, membrane integral proteins, cytoplasmic viscosity, and surface-volume ratio. It largely decreases under hematologic disorders, including malaria, sickle cell anemia, sepsis, spherocytosis, and diabetes. $^{2}$

The malaria parasite Plasmodium falciparum (Pf) changes the structure and mechanical properties of RBCs when the parasite progresses from ring stage to schizont. These changes substantially decrease deformability and increase cytoadherence of Pf-infected RBCs to the vascular endothelium, eventually leading to flow occlusions of capillary vessels. ${ }^{3,4}$ Microscopic examination of Giemsa-stained blood smear has been used as a standard method to diagnose malaria. Although this technique can identify parasitemia as low as $0.001 \%, 5$ it has several disadvantages, such as extremely low throughput, tedious operation procedure, and limited availability of experienced experts. Recently, various microfluidic devices have been suggested as an ideal platform to detect Pf-infected RBCs effectively.

As changes in hemorheological or hemodynamic properties are strongly related to hematological diseases, various blood biophysical properties, including viscosity, erythrocyte sedimentation rate, ${ }^{6}$ and deformability, have been used to detect Pf-infected RBCs. Several experimental techniques that utilize multiple physical principles, including hydrodynamics, optics, and electromagnetics, have been proposed to diagnose Pfinfected RBCs. First, morphological deformation of a single $\mathrm{RBC}$ that passes through a straight or constricted microfluidic channel is carefully monitored. Various biophysical properties, such as minimum cylinder diameter, ${ }^{7}$ transit time, ${ }^{8}$ occlusion, ${ }^{9}$

Received: December 24, 2015

Accepted: February 4, 2016

Published: February 4, 2016 
A

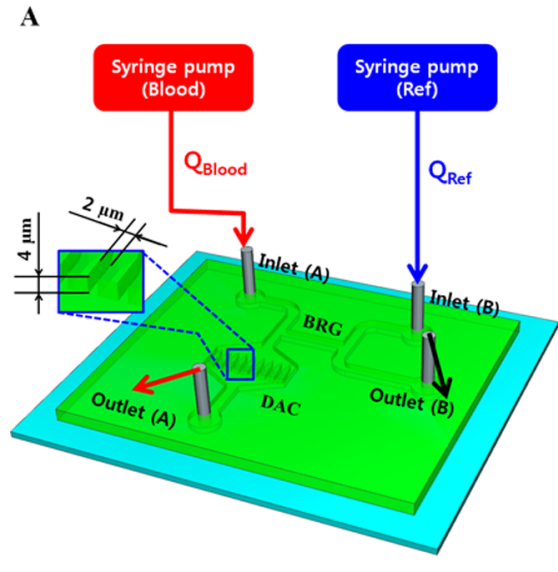

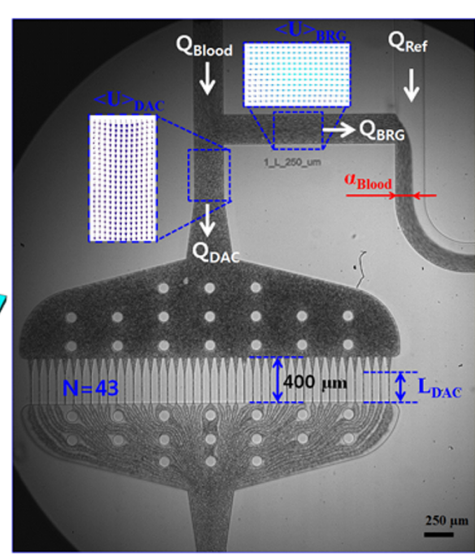

$\mathbf{D}_{(\mathbf{a})}$

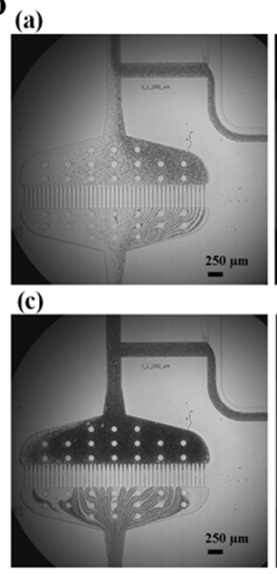

(b)

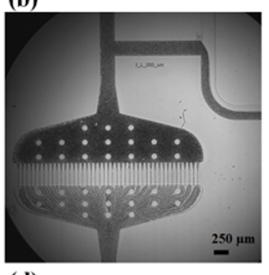

(d)

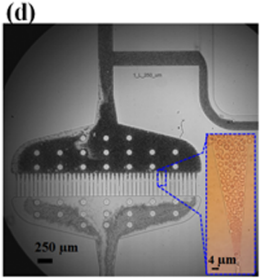

B

(a) $T=t_{1}$

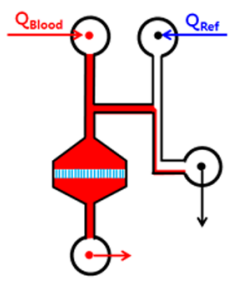

(b) $T=t_{2}$

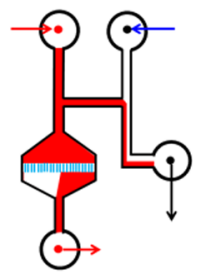

(c) $T=t_{3}$

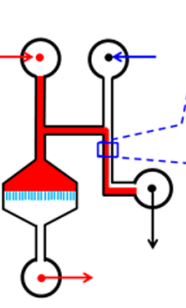

(d) $T>t_{3}$

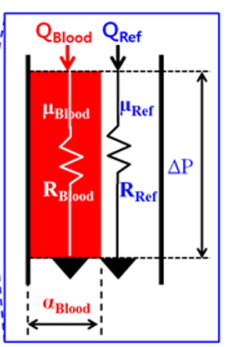

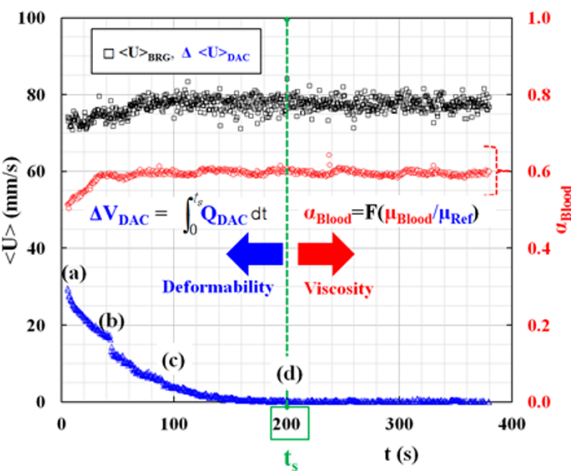

Figure 1. Schematics of the proposed method for simultaneous measurement of the deformability and viscosity of blood samples by monitoring hemodynamic variations in the microfluidic device. (A) Schematic of the experimental setup, including two syringe pumps for delivering the blood sample and reference fluid, and a disposable microfluidic device for measuring deformability and viscosity of the blood sample. The microfluidic device is conceptually mimicked from a Wheatstone-bridge electric circuit. It consists of two inlets and outlets, two identical upper channels, two lower side channels, and one bridge channel (BRG). The left lower side channel has a deformability assessment chamber (DAC) with parallel microfluidic filters (gap $=2 \mu \mathrm{m}$, depth $=4 \mu \mathrm{m}$, length $=L_{\mathrm{DAC}}$, and $N=43$ ), which is specially devised to measure RBC deformability. (B) The working principle for measuring the deformability and viscosity of blood samples. When blood sample and reference fluid are supplied into each inlet, all channels except the right upper side channel are filled with blood sample. After a certain period from $(\mathrm{a}) T=t_{1}$ to (b) $T=t_{2}$, the microfluidic filters in the DAC are partially clogged because of RBC deformability. At (c) $T=t_{3}$, most of the microfluidic filters are clogged. (d) At $T$ $>t_{3}$, the blood sample and reference fluid are supplied into the right lower side channel. The blood-filled width $\left(\alpha_{\text {Blood }}\right)$ varies depending on viscosity ratio $\left(\mu_{\text {Blood }} / \mu_{\text {Ref }}\right)$ and flow-rate ratio $\left(Q_{\text {Blood }} / Q_{\text {Ref }}\right)$. (C) Average velocities of blood flow in the DAC $\left(\langle U\rangle_{\text {DAC }}\right)$ and bridge channel $\left(\langle U\rangle_{\text {BRG }}\right)$ are evaluated using a time-resolved micro-PIV technique. At intervals of $0.5 \mathrm{~s}$, two microscopic images are consecutively captured with a high-speed camera at a frame rate of $5 \mathrm{kHz}$. (D) For a preliminary demonstration, blood sample $\left(H_{c t}=50 \%\right)$ is prepared by adding normal RBCs into $1 \times$ PBS solution. In this study, $1 \times$ PBS solution is used as the reference fluid. Snapshots are captured at different time points $(t)[(\mathrm{a}) t=0 \mathrm{~s}$, (b) $t=20 \mathrm{~s},(\mathrm{c})$ $t=100 \mathrm{~s}$, and (d) $t=200 \mathrm{~s}]$. By conducting a time-resolved micro-PIV technique and digital image processing, the average velocities of blood flow in the DAC and bridge channel $\left(\langle U\rangle_{\mathrm{DAC}},\langle U\rangle_{\mathrm{BRG}}\right)$ and blood-filled width $\left(\alpha_{\mathrm{Blood}}\right)$ are obtained as a function of time. $\langle U\rangle_{\mathrm{DAC}}$ gradually decreases because of consecutive clogging of RBCs in the microfluidic filters. RBC deformability is quantified by integrating the flow rate in the DAC for $200 \mathrm{~s}$. After $200 \mathrm{~s}$, the viscosity of blood sample is evaluated by monitoring the blood-filled width $\left(\alpha_{\text {Blood }}\right)$, which remains constant.

RBC velocity, ${ }^{10}$ lateral migration, ${ }^{11}$ cortical tension, ${ }^{12}$ electrical impedance, ${ }^{13}$ and cytoadherence, ${ }^{14}$ for individual Pf-infected RBCs exhibit different values, compared with those for normal RBCs. Second, under quasi-static flow conditions, morphological properties, such as membrane fluctuations ${ }^{15}$ and optical stretcher-based elasticity, ${ }^{16}$ are used to quantify Pf-infected RBCs. Finally, hemozoin, which is converted from hemoglobin during parasite progression, shows variation of magnetic characteristics. ${ }^{17}$ Its lateral migration is evaluated to detect Pfinfected RBCs under a magnetic field. Most of these previous methods have been employed to detect individual Pf-infected RBCs by measuring RBC deformability. However, RBC deformability changes under various pathological conditions. Therefore, additional quantification of biophysical properties of a blood sample is required to detect Pf-infected RBCs effectively. In addition, these single RBC-based diagnostic methods have limitations in practical applications, especially from extremely low throughput. Thus, a new microfluidic-based method that can provide high throughput is necessary.

In this study, a simple, high-throughput, and label-free measurement technique is proposed to detect Pf-infected RBCs through quantitative measurement of hemodynamic variations in a microfluidic analogue of a Wheatstone-bridge electric circuit. The microfluidic device has two inlets and outlets, as well as a typical H-shaped channel with a deformability assessment chamber (DAC). The DAC is composed of parallel microfluidic filters with a microsized gap. After injecting blood sample and reference fluid into each inlet of the microfluidic device, hemodynamic variations in the microfluidic channels are measured using a time-resolved microparticle image velocimetry (PIV) technique. First, the deformability of blood samples is evaluated by analyzing blood flow in the DAC for a certain period. The deformability of blood samples can be measured with high throughput and subpopulation measurement 
accuracy. Using the parallel flow formed by the two fluids on the right lower side channel of the microfluidic device, the viscosity ratio of blood sample to the reference fluid is measured by monitoring the blood-filled width and using hydrodynamic force balance in the channel. Thus, both deformability and blood-filled width in parallel flow are used to detect Pf-infected RBCs via a label-free and high-throughput technique.

Compared with previous measurement techniques, the proposed method has distinct advantages. First, the proposed method can evaluate the RBC deformability of a small amount of blood sample $(\sim 0.2 \mathrm{~mL})$ with a high hematocrit $(\sim 50 \%)$, especially within a short time period $(\sim 5 \mathrm{~min})$. Second, it can detect small variations in RBC deformability caused by subpopulations in heterogeneous blood sample with high sensitivity. Third, the proposed method can measure blood viscosity as a hemorheological property that depends on several factors, including hematocrit, deformability, aggregation, and base solution. Finally, this technique can quantitatively evaluate the cytoadherence of Pf-infected RBCs by measuring the temporal variations in velocity fields of the flow of the blood sample passing through the microfluidic channel.

The performance of the proposed method is quantitatively evaluated by varying four factors, namely, the size of microfluidic filter in the DAC, flow rate and hematocrit of the blood sample, and base solution. Thereafter, as a preliminary demonstration for the diagnosis of malaria-infected blood sample with low parasitemia, human blood samples that are partially mixed with normal RBCs and hardened RBCs are tested to quantitatively evaluate variations in RBC deformability caused by small differences in subpopulations. In addition, change of blood flow due to enhanced RBC aggregation is evaluated. Finally, as a clinical application, the proposed method is applied to detect Pf-infected RBCs.

\section{EXPERIMENTAL SECTION}

Simultaneous Measurement of Deformability and Viscosity of Blood Samples. A simple, label-free, and highthroughput measurement technique is proposed to detect Pfinfected RBCs. This method can measure the deformability and viscosity of blood samples by monitoring hemodynamic variations in a microfluidic analogue of a Wheatstone-bridge electric circuit. In addition, the proposed method can precisely detect small differences in subpopulations by measuring velocity fields of blood flow in the microfluidic channel.

The experimental setup is composed of two syringe pumps, a microfluidic device for measuring deformability and viscosity of blood sample, and an image acquisition system composed of a microscope and high-speed camera. As shown in Figure 1A, the microfluidic device is conceptually mimicked from a Wheatstone-bridge electric circuit. The device consists of two inlets and outlets, two identical upper channels, a left lower side channel with a DAC, right lower side channel, and one bridge channel (BRG). The DAC has parallel microfluidic filters (width $=2 \mu \mathrm{m}$, depth $=4 \mu \mathrm{m}$, length $=L_{\mathrm{DAC}}$, and $N=43$ ). Each disposable syringe is filled with an equal volume $(\sim 0.2$ $\mathrm{mL}$ ) of blood sample and reference fluid. After installing the two syringes into each syringe pump, the blood sample (i.e., Blood) and reference fluid (i.e., Ref) are supplied into the microfluidic device at a fixed flow rate of $1 \mathrm{~mL} / \mathrm{h}\left(Q_{\text {Blood }}=Q_{\text {Ref }}\right.$ $=1 \mathrm{~mL} / \mathrm{h}$ ).

As shown in Figure 1B, the deformability and viscosity of blood sample are sequentially measured by monitoring hemodynamic variations in the DAC and right lower side channel. Two fluids are supplied into the microfluidic device at the same flow rate $\left(Q_{\text {Blood }}=Q_{\text {Ref }}\right)$. Therefore, all channels except the right upper side channel are filled with blood sample because the viscosity of blood sample is greater than that of the reference fluid, and the overall fluidic resistance in the DAC is much greater than that in the right lower side channel. Instead, the upper right side channel is only filled with the reference fluid.

At $T=t_{1}$, all microfluidic filters in the DAC are completely filled with the blood sample as shown in Figure 1B-(a). After a certain time is elapsed from $T=t_{1}$ [Figure $\left.1 \mathrm{~B}-(\mathrm{a})\right]$ to $T=t_{2}$ [Figure 1B-(b)], the microfluidic filters in the DAC are partially clogged because of RBC deformability, which hinders blood flow in the DAC. At $T=t_{3}$ [Figure 1B-(c)], the microfluidic filters in the DAC are completely clogged. Blood flow in the DAC is totally interrupted. Moreover, the lower regions of the DAC are only filled with base solution of the blood sample. Thereafter, at $T>t_{3}$ [Figure 1B-(d)], blood sample and reference fluid are separated, and formed as parallel flows into the right lower side channel. Using a discrete parameter model, the fluidic circuit is simply modeled with flow rate $\left(Q_{\text {Blood }}, Q_{\text {Ref }}\right)$ and fluidic resistance $\left(R_{\text {Blood }}, R_{\text {Ref }}\right)$. For a rectangular channel ( $w=$ width, $h=$ depth) with an extremely low aspect ratio (i.e., $h / w=4 / 250)$, fluidic resistance $\left(R_{\mathrm{f}}\right)$ is approximately derived as $R_{\mathrm{f}}=12 \mu L /\left(w h^{3}\right)$. Since depth $(h)$ and length $(L)$ of the microfluidic channel are the same for both fluids, fluidic resistance ratio (i.e., $R_{\mathrm{Blood}} / R_{\mathrm{Ref}}$ ) is mathematically derived as $R_{\text {Blood }} / R_{\text {Ref }}=\left(\mu_{\text {Blood }} / \mu_{\text {Ref }}\right) \cdot\left(1-\alpha_{\text {Blood }}\right) / \alpha_{\text {Blood }}$. Here, $\mu_{\text {Blood }}$ and $\mu_{\text {Ref }}$ represent the viscosities of blood sample and reference fluid, respectively. In addition, taking into account the fact that each fluid has the same pressure drop in the channel (i.e., $\Delta P=$ $R_{\text {Blood }} \cdot Q_{\text {Blood }}=R_{\text {Ref }} Q_{\text {ref }}$ ), viscosity ratio (i.e., $\mu_{\text {Blood }} / \mu_{\text {Ref }}$ ) is simply derived as eq 1

$$
\frac{\mu_{\text {Blood }}}{\mu_{\text {Ref }}}=\left(\frac{\alpha_{\text {Blood }}}{1-\alpha_{\text {Blood }}}\right)\left(\frac{Q_{\text {Ref }}}{Q_{\text {Blood }}}\right)
$$

This equation indicates that the blood-filled width $\left(\alpha_{\text {Blood }}\right)$ varies depending on the viscosity ratio $\left(\mu_{\text {Blood }} / \mu_{\text {Ref }}\right)$ at the same flow rate condition (i.e., $Q_{\text {Blood }}=Q_{\text {Ref }}$ ).

Flow rates in the DAC $\left(Q_{\mathrm{DAC}}\right)$ and bridge channel $\left(Q_{\mathrm{BRG}}\right)$ are needed to measure the deformability and viscosity of the blood sample. As shown in Figure 1C, velocity fields of the blood sample in the DAC and bridge channel are measured using a time-resolved micro-PIV technique. Average velocities of the blood sample in the DAC $\left(\langle U\rangle_{\text {DAC }}\right)$ and bridge channel $\left.(<U\rangle_{\text {BRG }}\right)$ are calculated using the arithmetic average method. As the blood sample is constantly supplied at a flow rate of

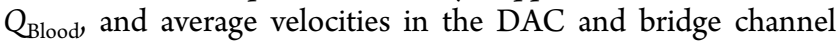
are estimated with respect to time, two flow rates $\left(Q_{D A C}\right.$ and $\left.Q_{B R G}\right)$ are mathematically evaluated as eqs 2 and 3

$$
\begin{aligned}
Q_{\mathrm{DAC}} & =\frac{\langle U\rangle_{\mathrm{DAC}}}{\langle U\rangle_{\mathrm{DAC}}+\langle U\rangle_{\mathrm{BRG}}} Q_{\mathrm{Blood}} \\
Q_{\mathrm{BRG}} & =\frac{\langle U\rangle_{\mathrm{BRG}}}{\langle U\rangle_{\mathrm{DAC}}+\langle U\rangle_{\mathrm{BRG}}} Q_{\mathrm{Blood}}
\end{aligned}
$$

In eq 3 , when $\langle U\rangle_{\text {DAC }} \approx 0, Q_{B R G}$ is equal to $Q_{\text {Blood }}$. Thus, all blood samples pass through the bridge channel without any loss, as shown in Figure 1B-(d). 
For a preliminary demonstration, a blood sample with a hematocrit of $H_{\mathrm{ct}}=50 \%$ is prepared by adding normal RBCs into $1 \times$ PBS solution. In addition, $1 \times$ PBS solution is used as the reference fluid. As shown in Figure 1D, microscopic images are consecutively captured at different lapses of time $(t)[(a) t=$ $5 \mathrm{~s}$, (b) $t=40 \mathrm{~s}$, (c) $t=100 \mathrm{~s}$, (d) $t=200 \mathrm{~s}$ ]. By conducting the time-resolved micro-PIV technique and digital image processing, the average velocities in the DAC and bridge channel $\left(\langle U\rangle_{\text {DAC }},\langle U\rangle_{\text {BRG }}\right)$ and blood-filled width $\left(\alpha_{\text {Blood }}\right)$ with respect to time are measured. From the preliminary demonstration, $\langle U\rangle_{\text {DAC }}$ is gradually decreased because of consecutive clogging of RBCs in the microfluidic filters of the DAC. After approximately $200 \mathrm{~s}$, the blood flow in the DAC is completely stopped (i.e., $\langle U\rangle_{\text {DAC }} \approx 0$ ). This step implies that the clogging of RBCs in the microfluidic filters is closely correlated with RBC deformability. RBC deformability can be quantified by integrating the temporal variations in the average velocity or flow rate for a certain period. As a new deformability index, the blood volume delivered into the DAC $\left(\Delta V_{\mathrm{DAC}}\right)$ is evaluated by integrating the flow rate in the DAC $\left(Q_{D A C}\right)$ for $200 \mathrm{~s}$ (i.e., $t_{s}=$ $200 \mathrm{~s})$ as eq 4

$$
\Delta V_{\mathrm{DAC}}=\int_{t=0}^{t=t_{s}} Q_{\mathrm{DAC}}(t) \mathrm{d} t
$$

Given that blood flow in the DAC is stopped by completely closing the outlet (A), all blood samples pass through the bridge channel (i.e., $Q_{B R G}=Q_{B l o o d}$ ). Using parallel flow in the right side channel, the viscosity variation of blood sample is evaluated by monitoring the blood-filled width $\left(\alpha_{\text {Blood }}\right)$. Furthermore, to quantify the variation of blood flow due to cytoadherence of RBCs in the microfluidic channel, the blood volume delivered into the bridge channel $\left(\Delta V_{\mathrm{BRG}}\right)$ is evaluated by integrating the flow rate in the bridge $\left(Q_{\mathrm{BRG}}\right)$ for $100 \mathrm{~s}$ using eq 5 ,

$$
\Delta V_{\mathrm{BRG}}=\int_{t=t_{0}}^{t=t_{0}+100 \mathrm{~s}} Q_{\mathrm{BRG}}(t) \mathrm{d} t
$$

Here, $t_{0}$ is greater than $200 \mathrm{~s}$ (i.e., $t_{0}>200 \mathrm{~s}$ ).

Fabrication of the Microfluidic Device and Experimental Procedure. The microfluidic device, which mimics a Wheatstone-bridge electric circuit, consists of two inlets, two outlets, and two parallel side channels. A bridge channel (length $=1500 \mu \mathrm{m}$, width $=250 \mu \mathrm{m})$ is connected to both side channels. The upper side channels have identical dimensions (length $=7300 \mu \mathrm{m}$, width $=250 \mu \mathrm{m})$. The left lower side channel is composed of parallel microfluidic filters $(N=43$, gap $=2 \mu \mathrm{m}$, overall length $=400 \mu \mathrm{m})$ in the DAC. The lower right side channel is $7300 \mu \mathrm{m}$ in length and $250 \mu \mathrm{m}$ in width. The depth of the microfluidic channels is fixed at $4 \mu \mathrm{m}$.

A silicon-master molder is fabricated using microelectromechanical system fabrication techniques, including photolithography and deep reactive ion etching. Thereafter, polydimethylsiloxane (PDMS) (Sylgard 184, Dow Corning, Midland, MI, U.S.A.) is mixed at a 1:10 ratio of curing agent to PDMS monomer. The PDMS mixture is then poured into the siliconmaster molder placed in a Petri dish. Air bubbles dissolved in PDMS are sufficiently removed using a vacuum pump. After curing PDMS in a convection oven at $80^{\circ} \mathrm{C}$ for $2 \mathrm{~h}$, the PDMS sheet is peeled off from the silicon-master molder. A PDMS block is cut from the PDMS sheet with a razor blade. Two inlets and two outlets of the PDMS block are punched with a biopsy punch (O.D. $=1 \mathrm{~mm})$. After treating the PDMS block and a glass slide with oxygen plasma, a microfluidic device is fabricated by bonding the PDMS block to the glass slide.

The microfluidic device is then placed on an optical microscope (Nikon, Tokyo, Japan) attached with a $4 \times$ objective lens $(\mathrm{NA}=0.1)$. Two disposable syringes $(1 \mathrm{~mL}$, BD Biosciences, Singapore) are filled with $1 \%$ bovine serum albumin (BSA) solution. Each disposable syringe is connected to the two inlets with a polyethylene tube (I.D. $=250 \mu \mathrm{m}$, length $=300 \mathrm{~mm}$ ). In addition, two polyethylene tubes (I.D. = $250 \mu \mathrm{m}$, length $=100 \mathrm{~mm}$ ) are connected to the two outlets. After installing two disposable syringes into a syringe pump (neMESYS, centoni GmbH, Germany), 1\% BSA solution is delivered into the microfluidic device at a flow rate of $1 \mathrm{~mL} / \mathrm{h}$ for $5 \mathrm{~min}$. Air bubbles in the microfluidic device are completely removed. Thereafter, the two disposable syringes are filled with the blood sample $\left(V_{\text {Blood }}=0.2 \mathrm{~mL}\right)$ and $1 \times$ PBS solution $\left(V_{\text {Ref }}\right.$ $=0.2 \mathrm{~mL}$ ). After installing two syringes into the syringe pumps, both fluids are delivered into the microfluidic device at the same flow rate of $1 \mathrm{~mL} / \mathrm{h}$ (i.e., $Q_{\text {Blood }}=Q_{\text {Ref }}=1 \mathrm{~mL} / \mathrm{h}$ ). All experiments are conducted at room temperature $\left(25^{\circ} \mathrm{C}\right)$.

Time-Resolved Micro-PIV Technique and Digital Image Processing. A high-speed camera (FASTCAM SA 1.1, Photron, San Diego, CA, U.S.A.) is employed to capture images of blood flow in the microfluidic channel. The camera has a spatial resolution of $1280 \times 1024$ pixels, with each pixel corresponding to $10 \mu \mathrm{m}$. For long-term measurement of $5 \mathrm{~min}$, the high-speed camera is synchronized using a digital delay pulse generator (Model 555, BNC, San Rafael, CA, U.S.A.). After setting the frame rate of the high-speed camera at $5 \mathrm{kHz}$, two consecutive images are sequentially captured at intervals of $0.5 \mathrm{~s}$. To evaluate the temporal hemodynamic variations in the DAC and bridge channel, velocity fields of blood flow in the DAC and bridge channel are quantified by conducting a timeresolved digital micro-PIV technique. Two specific regions of interest (ROIs) measuring $50 \times 60$ pixels in the DAC and bridge channel are selected to obtain velocity fields of blood flow. The interrogation window size is $8 \times 8$ pixels, and window overlap is $50 \%$. The obtained velocity vectors are validated with a median filter. By obtaining the arithmetic average of instantaneous two velocity fields, the average velocities in the DAC and BRG $\left(\langle U\rangle_{\text {DAC }},\langle U\rangle_{\text {BRG }}\right)$ with respect to time are obtained. In addition, a specific ROI $(50 \times 50$ pixels $)$ in the lower right side channel is carefully selected to evaluate the blood-filled width $\left(\alpha_{\text {Blood }}\right)$. Using a commercial software (Matlab, Mathworks, U.S.A.), temporal variations in the blood-filled width $\left(\alpha_{\text {Blood }}\right)$ in the right lower side channel are quantitatively evaluated from the images captured consecutively by the high-speed camera. An adaptive equal histogram method is used to enhance image contrast of the captured images. To obtain the interfacial position between the blood sample and reference fluid along the microfluidic channel, the captured images are converted into binary images by adopting Otsu's method. After obtaining the arithmetic average of pixel values in the ROI for each image, the blood-filled width ( $\left.\alpha_{\text {Blood }}\right)$ with respect to time is obtained.

Sample Preparation of Normal Blood and MalariaInfected Blood. The human RBCs and plasma tested in this study are provided by Daegu-Gyeongbuk Blood Bank (Gyeongbuk, South Korea). The hematocrit of the blood samples is adjusted to $30 \%, 40 \%$, and $50 \%$ by carefully adding normal RBCs into base solution [1× PBS solution $(\mathrm{pH} 7.4$, Bio Solution, Seoul, South Korea), plasma]. To decrease the deformability of normal RBCs, normal RBCs are dipped into 
A

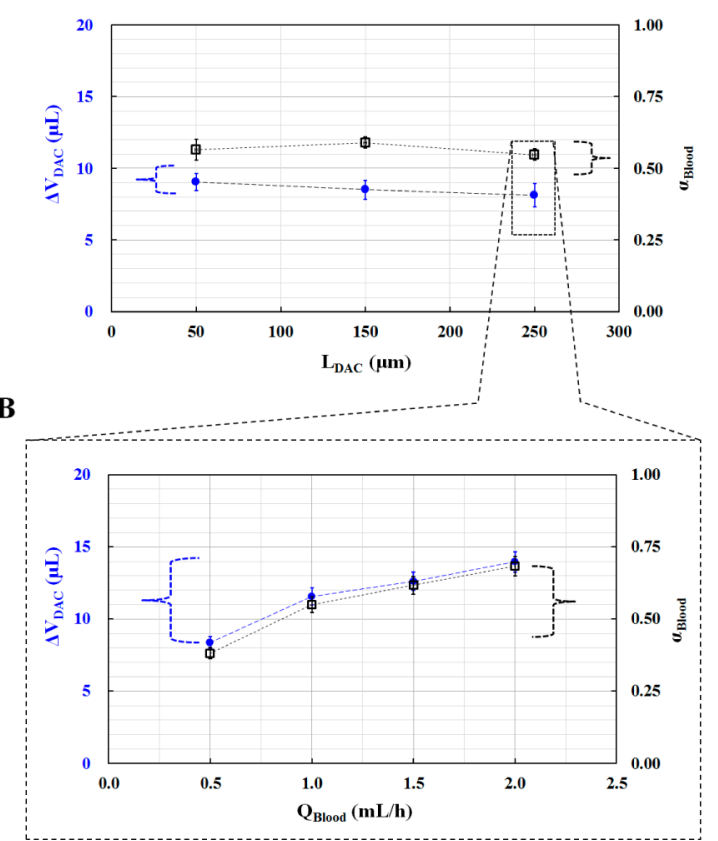

C

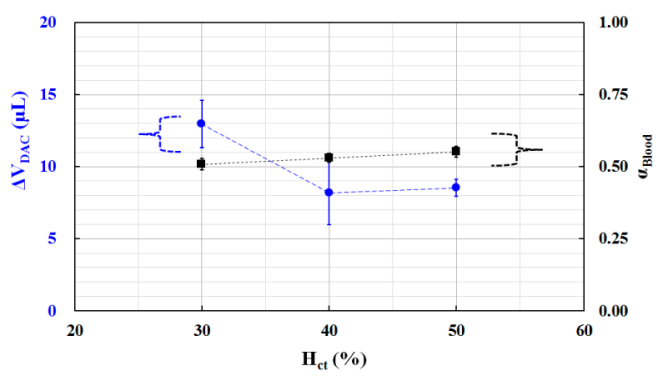

D

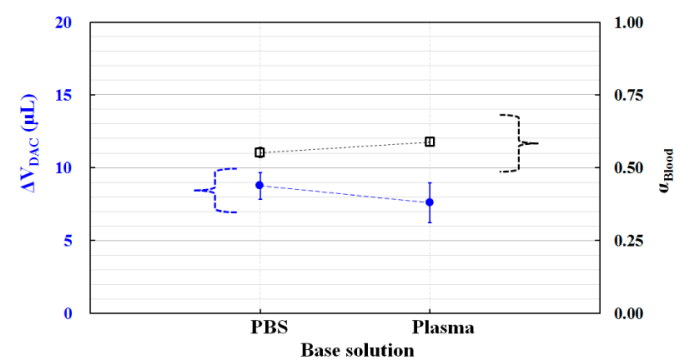

Figure 2. Quantitative evaluation on the effects of four factors, namely, length of microfluidic filter $\left(L_{\mathrm{DAC}}\right)$, blood flow rate $\left(Q_{\mathrm{Blood}}\right)$, hematocrit $\left(H_{\mathrm{ct}}\right)$, and base solution, on the blood volume delivered into the DAC $\left(\Delta V_{\text {DAC }}\right)$ and blood-filled width $\left(\alpha_{\text {Blood }}\right)$. (A) Variations in the delivered blood volume $\left(\Delta V_{\mathrm{DAC}}\right)$ and blood-filled width $\left(\alpha_{\text {Blood }}\right)$ with respect to the length of the microfluidic filter $\left(L_{\mathrm{DAC}}\right)\left[(\mathrm{a}) L_{\mathrm{DAC}}=50 \mu \mathrm{m},(\mathrm{b}) L_{\mathrm{DAC}}=150 \mu \mathrm{m}\right.$, and (c) $\left.L_{\mathrm{DAC}}=250 \mu \mathrm{m}\right]$. The length of the microfluidic filters is fixed at $250 \mu \mathrm{m}$ (i.e., $L_{\mathrm{DAC}}=250 \mu \mathrm{m}$ ). (B) Variations in $\Delta V_{\mathrm{DAC}}$ and $\alpha_{\mathrm{Blood}}$ with increasing flow rate of blood ( $\left.Q_{\text {Blood }}\right)\left[(\mathrm{a}) Q_{\text {Blood }}=0.5 \mathrm{~mL} / \mathrm{h},(\mathrm{b}) Q_{\text {Blood }}=1 \mathrm{~mL} / \mathrm{h},(\mathrm{c}) Q_{\text {Blood }}=1.5 \mathrm{~mL} / \mathrm{h}\right.$, and (d) $\left.Q_{\text {Blood }}=2 \mathrm{~mL} / \mathrm{h}\right]$. (C) Variations in $\Delta V_{\text {DAC }}$ and $\alpha_{\text {Blood }}$ with respect to hematocrit $\left(H_{\mathrm{ct}}\right)\left[(\mathrm{a}) H_{\mathrm{ct}}=30 \%\right.$, (b) $H_{\mathrm{ct}}=40 \%$, and (c) $\left.H_{\mathrm{ct}}=50 \%\right]$. (D) Variations in $\Delta V_{\mathrm{DAC}}$ and $\alpha_{\mathrm{Blood}}$ for two base solutions, such as plasma and $1 \times$ PBS solution.

various concentration of glutaraldehyde solution $\left(C_{\mathrm{GA}}\right)$ (Junsei Chemical Co., Tokyo, Japan), which ranges from $C_{\mathrm{GA}}=0.0 \%$ to $C_{\mathrm{GA}}=0.25 \%$ for $10 \mathrm{~min}$. The hematocrit of the hardened blood sample is adjusted to $50 \%$ by adding hardened RBCs into $1 \times$ PBS solution. The hematocrit of normal blood is also set to $50 \%$ by adding normal RBCs into $1 \times$ PBS solution. Heterogeneous blood samples with a hardened blood volume fraction $(\psi)[(\mathrm{a}) \psi=0 \%$, (b) $\psi=2 \%$, (c) $\psi=5 \%$, (d) $\psi=$ $10 \%$ ] are prepared by mixing hardened blood sample with normal blood sample. The hardened RBCs were prepared with GA solution of $0.25 \%$. In this study, $\psi=0 \%$ represents the normal blood sample.

Pf (isolate 3D7) is maintained in an incubator containing human RBCs (blood type O+), RPMI 1640 (buffered with 25 $\mathrm{mM}$ HEPES and $\left.25 \mathrm{mM} \mathrm{NaHCO}{ }_{3}\right), 10 \mu \mathrm{g} / \mathrm{mL}$ gentamycin, and $370 \mu \mathrm{M}$ hypoxanthine under a gas mixture $\left(5 \% \mathrm{CO}_{2}, 5 \%\right.$ $\mathrm{O}_{2}$, and $90 \% \mathrm{~N}_{2}$ ) at $37{ }^{\circ} \mathrm{C}$. The parasites are diluted using a culture medium containing normal RBCs to prepare blood samples with $5 \%$ hematocrit and maintain the parasitemia $(P)$ [(a) $P=0.0004 \%$, (b) $P=0.0045 \%$, (c) $P=0.0458 \%$, and (d) $P$ $=0.458 \%$. The hematocrit of the Pf-infected blood sample is set to $50 \%$ by adjusting with $1 \times$ PBS solution. In addition, to visualize the cytoadherence of Pf-infected RBCs to the microfluidic channel, a malaria-infected blood sample with parasitemia level of $1.9 \%$ is prepared to have a hematocrit of $5 \%$.

Giemsa Staining for Parasitemia Level Detection. A thin blood smear is fixed with $99 \%$ methanol for $5 \mathrm{~min}$. After fixing a slide, the slide is dried for 1-2 min. A diluted Giemsa stain solution (Merck, Darmstadt, Germany) is prepared prior to staining. The slide is immersed in freshly prepared Giemsa stain solution for $25 \mathrm{~min}$, rinsed with tap water, and dried. The stained malaria parasites are examined using a microscope (Zeiss, Jena, Germany) with a $100 \times$ oil immersion objective lens.

\section{RESULTS AND DISCUSSION}

Effect of Parameters on the Delivered Blood Volume. The blood volume delivered into the $\mathrm{DAC}\left(\Delta V_{\mathrm{DAC}}\right)$ is suggested as a deformability index to quantify the deformability of blood samples. In addition, the viscosity ratio of blood sample in relation to reference fluid is simultaneously monitored by detecting the blood-filled width $\left(\alpha_{\text {Blood }}\right)$ in the right lower side channel. $\Delta V_{\mathrm{DAC}}$ varies depending on overall fluidic resistance in the DAC, which is strongly influenced by the fluidic resistance in the parallel microfluidic filters. For example, when delivering blood sample with hardened RBCs, the RBCs are clogged or trapped in the microfluidic filters. This increased fluidic resistance causes to decrease the velocity of blood flow, which in turn decreases the blood volume delivered into the DAC. In addition, the blood-filled width $\left(\alpha_{\text {Blood }}\right)$ is monitored to detect variations in blood viscosity. As shown in Figure 2, the effects of several parameters, including length of microfluidic filter $\left(L_{\mathrm{DAC}}\right)$, blood flow rate $\left(Q_{\text {Blood }}\right)$, hematocrit $\left(H_{\mathrm{ct}}\right)$, and base solution, especially on the variations in $\Delta V_{\mathrm{DAC}}$ and $\alpha_{\text {Blood }}$ are experimentally investigated.

First, to evaluate the effect of the length of the microfluidic filter on the variations in $\Delta V_{\mathrm{DAC}}$ and $\alpha_{\text {Blood }}$ the blood sample and reference fluid were simultaneously supplied into the microfluidic device. The hematocrit of blood sample (normal RBCs in PBS suspension) was fixed at $50 \%$. In addition, a microfluidic device was designed to have different lengths of the microfluidic filter $\left(L_{\mathrm{DAC}}\right)\left[(\mathrm{a}) L_{\mathrm{DAC}}=50 \mu \mathrm{m},(\mathrm{b}) L_{\mathrm{DAC}}=150\right.$ $\mu \mathrm{m}$, and (c) $\left.L_{\mathrm{DAC}}=250 \mu \mathrm{m}\right]$. As shown in Figure $2 \mathrm{~A}, \Delta V_{\mathrm{DAC}}$ 
A

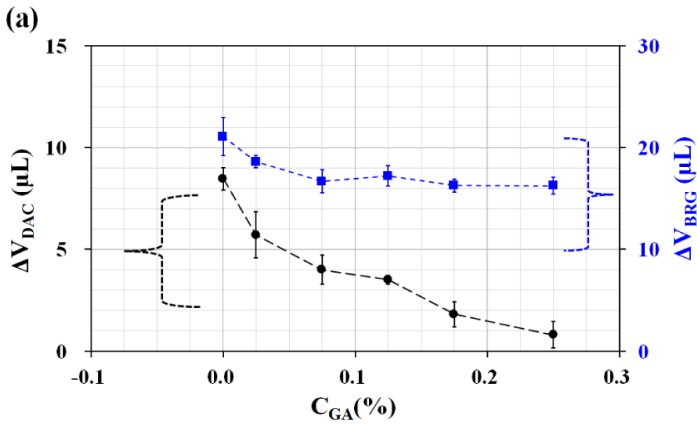

B

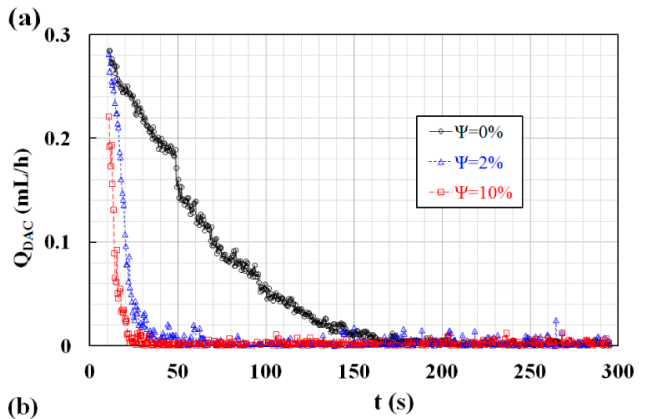

(b)

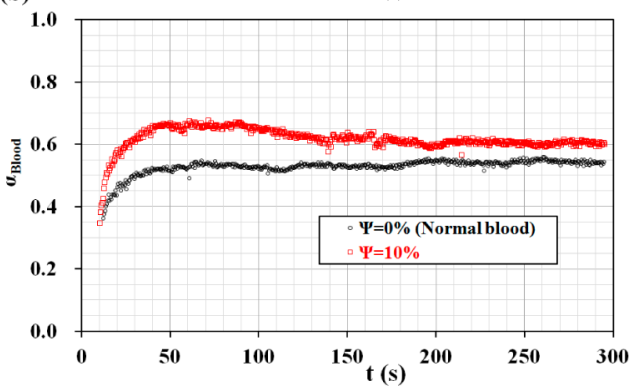

(b)

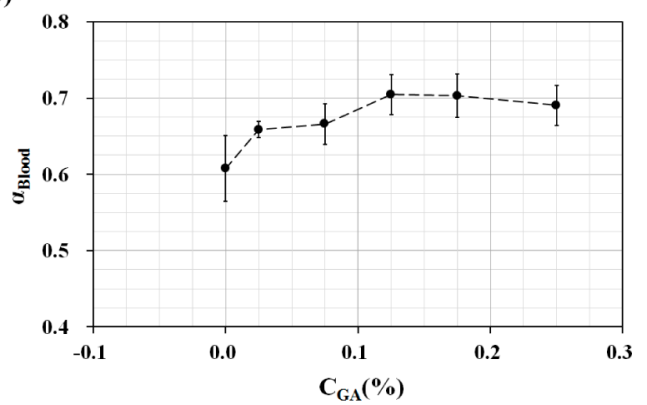

(c)
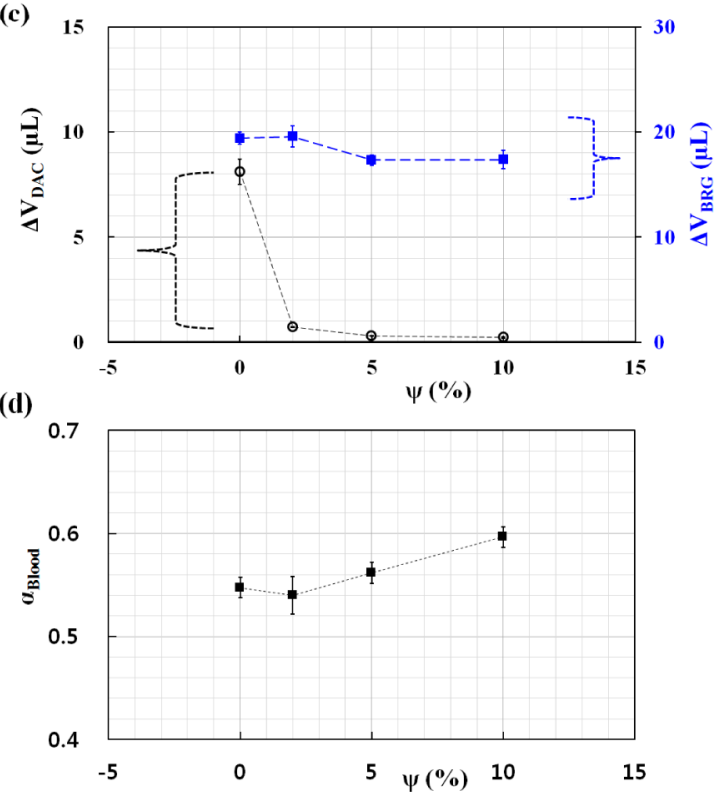

Figure 3. Quantitative performance evaluation of the proposed method for homogeneous and heterogeneous blood samples. (A) Performance evaluation for blood sample composed of homogeneous $\mathrm{RBCs}$ fixed with various concentrations of $\mathrm{GA}$ solution $\left(C_{\mathrm{GA}}\right)$ ranging from $C_{\mathrm{GA}}=0.0 \%$ to $C_{\mathrm{GA}}=0.25 \%$. (a) Variations of $\Delta V_{\mathrm{DAC}}$ and $\Delta V_{\mathrm{BRG}}$ with respect to $C_{\mathrm{GA}}$. (b) Variations of blood-filled width $\left(\alpha_{\mathrm{Blood}}\right)$ with respect to $C_{\mathrm{GA}}$. (B) Performance evaluation for blood sample prepared by adding hardened RBCs into normal RBCs. Here, the hardened RBCs were fixed only with GA solution of $0.25 \%$ (i.e., $C_{\mathrm{GA}}=0.25 \%$ ). (a) Temporal variations of flow rate of blood sample delivered into the DAC ( $\left.Q_{\mathrm{DAC}}\right)$ with respect to volume fraction of hardened blood $(\psi)$. The volume fraction is defined as the volume ratio of the hardened blood sample to the total blood sample. (b) Temporal variations of blood-filled width $\left(\alpha_{\text {Blood }}\right)$ for the normal blood sample $(\psi=0)$ and hardened blood sample $(\psi=10 \%)$. (c) Variations of $\Delta V_{\mathrm{DAC}}$ and $\Delta V_{\mathrm{BRG}}$ with respect to $\psi$. (d) Variations of blood-filled width $\left(\alpha_{\text {Blood }}\right)$ with respect to $\psi$.

and $\alpha_{\text {Blood }}$ were measured by varying the length of the microfluidic filter. As a result, the blood volume delivered into the DAC decreased with increasing length of the microfluidic filter. Given that the flow rate of blood in the DAC $\left(Q_{D A C}\right)$ is inversely proportional to the fluidic resistance caused by the microfluidic filter, $\Delta V_{\text {DAC }}$ was largely influenced by the length of the microfluidic filter. After closing the outlet (A), the bloodfilled width $\left(\alpha_{\text {Blood }}\right)$ was also measured with varying lengths of the microfluidic filter. As expected, $\alpha_{\text {Blood }}$ remained constant regardless of the length of the microfluidic filter. This finding implied that the blood sample was consistently delivered into the microfluidic device, and the length of the microfluidic filter must be fixed to effectively monitor variations in $\Delta V_{\text {DAC }}$ as a deformability index. Thus, the length of the microfluidic filter was fixed at $250 \mu \mathrm{m}$ (i.e., $L_{\text {DAC }}=250 \mu \mathrm{m}$ ).

Second, to evaluate the effect of blood flow rate $\left(Q_{B l o o d}\right)$ on $\Delta V_{\mathrm{DAC}}$ and $\alpha_{\text {Blood }}$ the flow rate of the reference fluid in the microfluidic device $\left(L_{\mathrm{DAC}}=250 \mu \mathrm{m}\right)$ was fixed at $1 \mathrm{~mL} / \mathrm{h}\left(Q_{\mathrm{Ref}}\right.$ $=1 \mathrm{~mL} / \mathrm{h}$ ). The hematocrit of blood sample was adjusted to $50 \%$ by carefully adding normal RBCs into $1 \times$ PBS solution. As shown in Figure 2B, $\Delta V_{\mathrm{DAC}}$ and $\alpha_{\text {Blood }}$ were measured with increasing blood flow rate $\left(Q_{\text {Blood }}\right)\left[(\mathrm{a}) Q_{\text {Blood }}=0.5 \mathrm{~mL} / \mathrm{h},(\mathrm{b})\right.$ $Q_{\text {Blood }}=1 \mathrm{~mL} / \mathrm{h}$, (c) $Q_{\text {Blood }}=1.5 \mathrm{~mL} / \mathrm{h}$, and $(\mathrm{d}) Q_{\text {Blood }}=2$ $\mathrm{mL} / \mathrm{h}]$. The results showed that $\Delta V_{\mathrm{DAC}}$ and $\alpha_{\text {Blood }}$ significantly increased with increasing blood flow rate. This finding suggested that the blood flow rate should be maintained constantly for consistent measurement of $\Delta V_{\mathrm{DAC}}$ and $\alpha_{\mathrm{Blood}}$. Therefore, the flow rates of the blood sample and reference fluid were maintained at the same flow rate of $1 \mathrm{~mL} / \mathrm{h}$ (i.e., $Q_{\text {Blood }}=Q_{\text {Ref }}=1 \mathrm{~mL} / \mathrm{h}$ ).

Third, to evaluate the effect of hematocrit $\left(H_{\mathrm{ct}}\right)$ on $\Delta V_{\mathrm{DAC}}$ and $\alpha_{\text {Blood }}$, three levels of hematocrit $\left(H_{\mathrm{ct}}\right)\left[(\mathrm{a}) H_{\mathrm{ct}}=30 \%\right.$, (b) $H_{c t}=40 \%$, and (c) $\left.H_{c t}=50 \%\right]$ were tested. The flow rates of the blood sample and reference fluid delivered into the microfluidic device $\left(L_{\mathrm{DAC}}=250 \mu \mathrm{m}\right)$ were fixed at $Q_{\text {Blood }}=$ $Q_{\text {Ref }}=1 \mathrm{~mL} / \mathrm{h}$. The variation in $\Delta V_{\mathrm{DAC}}$ and $\alpha_{\text {Blood }}$ with respect to hematocrit $\left(H_{\mathrm{ct}}\right)$ is shown in Figure $2 \mathrm{C}$. The value of $\Delta V_{\mathrm{DAC}}$ decreased up to $40 \%$ hematocrit. Thereafter, $\Delta V_{\text {DAC }}$ did not show significant variation with respect to the hematocrit level. In addition, $\alpha_{\text {Blood }}$ gradually increased with increasing 
A

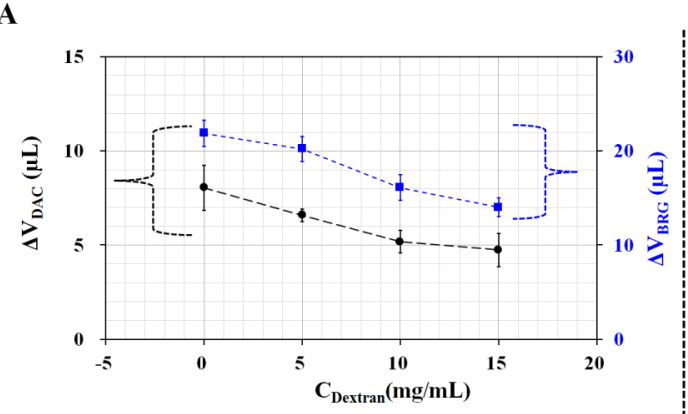

C

(a)

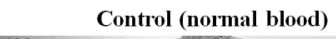

Dextran $(10 \mathrm{mg} / \mathrm{mL})$
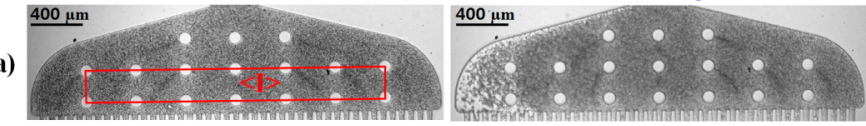

(b)

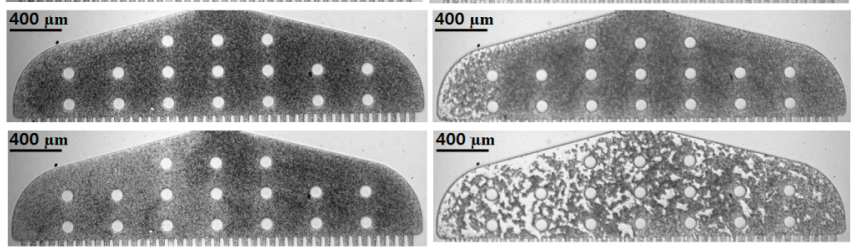

B
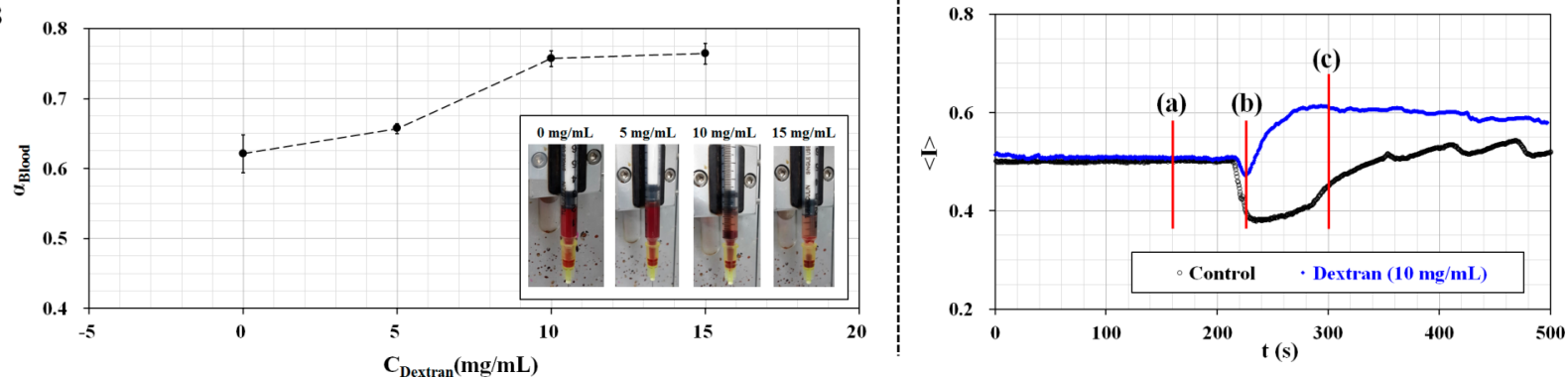

Figure 4. Quantitative performance evaluation for blood samples composed of enhanced RBC aggregation by adding Dextran solution. RBC aggregation was enhanced by adding different concentrations of Dextran solution $\left(C_{\text {Dextran }}\right)$ ranging from $C_{\text {Dextran }}=0 \mathrm{mg} / \mathrm{mL}$ to $C_{\text {Dextran }}=15 \mathrm{mg} / \mathrm{mL}$ into normal RBCs in PBS suspension (i.e., control blood sample). (A) Variations of $\Delta V_{\text {DAC }}$ and $\Delta V_{\text {BRG }}$ with respect to $C_{\text {Dextran. }}$ (B) Variations of $\alpha_{\text {Blood }}$ with respect to $C_{\text {Dextran }}$. Inset shows variations of RBCs-depleted regions in the vertically installed syringe for each concentration of Dextran solution after finishing the experiments. (C) Temporal variations of image intensity $(<I>)$ in a specific region of interest (ROI) in the upper region of the DAC.

hematocrit level. This finding implied that blood viscosity increased with increasing hematocrit level. Therefore, the variation in hematocrit was monitored by quantifying the blood-filled width $\left(\alpha_{\text {Blood }}\right)$. On the basis of this finding, the hematocrit level should be fixed at a constant value to minimize the effect of hematocrit variation. Thus, in this study, the hematocrit level of blood was fixed at 50\%.

Finally, the effect of base solution on the variations in $\Delta V_{\mathrm{DAC}}$ and $\alpha_{\text {Blood }}$ was tested. The hematocrit levels of two different blood samples were adjusted to $50 \%$ by adding normal RBCs into plasma or $1 \times$ PBS solution. The blood sample and reference fluid were supplied into the microfluidic device $\left(L_{\mathrm{DAC}}\right.$ $=250 \mu \mathrm{m})$ at the same flow rate of $Q_{\text {Blood }}=Q_{\text {Ref }}=1 \mathrm{~mL} / \mathrm{h}$. Variations in $\Delta V_{\mathrm{DAC}}$ and $\alpha_{\mathrm{Blood}}$ with respect to the base solution are shown in Figure 2D. The results showed that $\Delta V_{\mathrm{DAC}}$ and $\alpha_{\text {Blood }}$ significantly changed depending on the base solution. Considering that the viscosity of plasma is higher than that of $1 \times$ PBS solution, $\Delta V_{\mathrm{DAC}}$ of plasma is lower than that of $1 \times$ PBS solution. In addition, $\alpha_{\text {Blood }}$ of plasma is much higher than that of $1 \times$ PBS solution. Thus, blood samples were prepared with the same base solution.

Detection of Homogeneous and Heterogeneous Hardened RBCs. First, the performance of the proposed method was evaluated for blood sample composed of homogeneous hardened RBCs. The hardened RBCs were prepared by dipping normal RBCs into various concentrations of GA solution $\left(C_{\mathrm{GA}}\right)$, which ranges from $C_{\mathrm{GA}}=0.0 \%$ to $C_{\mathrm{GA}}=$ $0.25 \%$. The hematocrit of the hardened blood sample was adjusted to $50 \%$ by adding hardened RBCs into $1 \times$ PBS solution. As shown in Figure 3A-(a), blood volume delivered into the DAC $\left(\Delta V_{\mathrm{DAC}}\right)$ tends to decrease with increasing the concentrations of GA solution. In other words, depending on the concentration of GA solution, the normal RBCs are gradually fixed, which hinders and interrupts blood flows in the
DAC. The $\Delta V_{\mathrm{DAC}}$ of the hardened RBCs with low concentration of GA solution (i.e., $C_{\mathrm{GA}}=0.025 \%$ ) is much smaller than that of normal RBCs. This result implies that the blood volume delivered into the $\mathrm{DAC}$ (i.e., $\Delta V_{\mathrm{DAC}}$ ) can be usefully used as a deformability index. However, the $\Delta V_{\mathrm{BRG}}$ remains constant, even at high concentration of the GA solution. This result indicates that the hardened RBCs do not change blood flow due to cytoadherence of RBCs in the microfluidic channel. In addition, as shown in Figure 3A-(b), $\alpha_{\text {Blood }}$ is slightly increased at high concentration of GA solution. Because the deformability of normal RBCs is largely decreased depending on the concentration of GA solution, viscosity of the blood sample composed of hardened RBCs increases, which causes an increase in the blood-filled width $\left(\alpha_{\text {Blood }}\right)$.

Then, as a preliminary demonstration for feasible diagnosis of Pf-infected RBCs with low parasitemia, blood samples partially mixed with normal and hardened RBCs were prepared to quantify the variations in RBC deformability and viscosity caused by small changes in subpopulations of RBCs. The hardened RBCs were prepared with GA solution of $0.25 \%$. The hematocrit of the hardened blood sample is adjusted to $50 \%$ by adding hardened RBCs into $1 \times$ PBS solution. To evaluate the performance of the proposed method, heterogeneous blood samples with different hardened blood volume fractions $(\psi)$ [(a) $\psi=0 \%$, (b) $\psi=2 \%$, (c) $\psi=5 \%$, and (d) $\psi=10 \%]$ were prepared by partially adding normal blood sample into the hardened blood sample. The volume faction $(\psi)$ is defined as the volume ratio of the hardened blood sample to the total blood sample (i.e., $\psi=V_{\text {Hardened Blood }} / V_{\text {Total Blood }}$ ). In this demonstration, the flow rates of blood sample and reference fluid were supplied into the microfluidic device $\left(L_{\mathrm{DAC}}=250\right.$ $\mu \mathrm{m}$ ) at the same flow rate of $1 \mathrm{~mL} / \mathrm{h}$ (i.e., $Q_{\text {Blood }}=Q_{\text {Ref }}=1$ $\mathrm{mL} / \mathrm{h})$. 
The temporal variations in flow rate of blood sample delivered into the DAC $\left(Q_{\mathrm{DAC}}\right)$ with respect to the hardened blood volume fraction $(\psi)[(\mathrm{a}) \psi=0 \%$, (b) $\psi=2 \%$, and (c) $\psi$ $=10 \%]$ are shown in Figure 3B-(a). The results showed that the flow rate of blood sample supplied into the DAC $\left(Q_{D A C}\right)$ dramatically decreased with increasing hardened blood volume fraction. Successive clogging of RBCs in the microfluidic filter, which contributed to increasing fluidic resistance in the DAC, was significantly enhanced at high values of the hardened blood volume fraction. Thus, the flow rate of blood sample delivered into the DAC decreased with increasing hardened blood volume fraction. The flow rate supplied into the DAC nearly stopped within $200 \mathrm{~s}$. After the blood flow stopped, all of the blood samples flowed into the right lower side channel. Therefore, variations in blood viscosity were directly quantified using eq 1 . The blood sample and reference fluid were supplied into the right lower side channel as parallel flow at the same flow rate of $Q_{\text {Blood }}=Q_{\text {Ref }}=1 \mathrm{~mL} / \mathrm{h}$.

As shown in Figure $3 \mathrm{~B}-(\mathrm{b})$, the temporal variations in the blood-filled width $\left(\alpha_{\text {Blood }}\right)$ for two different blood samples [(a) $\psi=0$, and (b) $\psi=10 \%$ ] were quantitatively compared. Bloodfilled width $\left(\alpha_{\text {Blood }}\right)$ was remained constant after 200 s. In addition, $\alpha_{\text {Blood }}$ of the hardened blood sample $(\psi=10 \%)$ was distinctively higher than that of the normal blood sample $(\psi=$ $0 \%)$. This finding indicates that blood viscosity of the hardened blood sample is higher than that of the normal blood sample. To quantify the variations in blood viscosity, the average value of $\alpha_{\text {Blood }}$ was obtained using several data $(n=100)$ after $200 \mathrm{~s}$

Using the temporal variations in $Q_{D A C}$ as shown in Figure 3B-(a) and eq 4, blood volume delivered into the DAC $\left(\Delta V_{\mathrm{DAC}}\right)$ was quantified with respect to the hardened blood volume fraction $(\psi)$. As depicted in Figure 3B-(c), $\Delta V_{\text {DAC }}$ decreased with increasing hardened blood volume fraction. In addition, the heterogeneous blood sample with $\psi=2 \%$ showed distinctly different values of $\Delta V_{\mathrm{DAC}}$ compared with normal blood sample $(\psi=0 \%)$. Thus, the blood volume delivered into the DAC $\left(\Delta V_{\text {DAC }}\right)$ could be suitably considered as a deformability index. In addition, $\Delta V_{\mathrm{BRG}}$ was slightly decreased after $\psi=2$. In other words, $\Delta V_{\mathrm{BRG}}$ was decreased because fluidic resistance (or fluid viscosity) was increased depending on the hardened volume fraction $(\psi)$. The blood viscosity was evaluated by monitoring the blood-filled width $\left(\alpha_{\text {Blood }}\right)$ with respect to the hardened blood volume fraction $(\psi)$. As shown in Figure 3B-(d), $\alpha_{\text {Blood }}$ did not show noticeable variation up to $\psi=5 \%$. However, $\alpha_{\text {Blood }}$ of heterogeneous blood sample with $\psi$ $=10 \%$ was higher than that of normal blood sample. From this result, the blood volume supplied into the DAC $\left(\Delta V_{\text {DAC }}\right)$ was more effective than the blood-filled width $\left(\alpha_{\mathrm{Blood}}\right)$ in the accurate detection of subpopulations in heterogeneous blood samples $(\psi<5 \%)$.

Detection of Enhanced Aggregation of RBCs. Alternation of blood flow due to enhanced aggregation of RBCs was evaluated by using the proposed method. RBC aggregation was gradually enhanced by adding normal RBCs into different concentrations of Dextran solution $\left(C_{\text {Dextran }}\right)(\mathrm{MW}=70 \mathrm{kDa}$, Sigma-Aldrich, U.S.A.) which ranges from $C_{\text {Dextran }}=0 \mathrm{mg} / \mathrm{mL}$ to $C_{\text {Dextran }}=15 \mathrm{mg} / \mathrm{mL}$. As shown in Figure $4 \mathrm{~A}$, the $\Delta V_{\mathrm{DAC}}$ and $\Delta V_{\mathrm{BRG}}$ are gradually decreased with increasing the concentrations of Dextran solution. In addition, the blood-filled width $\left(\alpha_{\text {Blood }}\right)$ is increased with increasing concentration of the Dextran solution as depicted in Figure 4B. Previous study on erythrocyte sedimentation rate (ESR) measurement reported that Dextran solution increases ESR in a driving syringe. ${ }^{6}$ The
RBCs-depleted region is expanded from the top with increasing concentration of Dextran solution. Inset of Figure 4B shows variations of RBCs-depleted region in a driving syringe after completing experiments. Since blood sample was supplied from the bottom position of the syringe into a microfluidic device, hematocrit of the blood sample supplied into the microfluidic device is increased with increasing concentration of Dextran solution. Thus, the blood-filled width $\left(\alpha_{\text {Blood }}\right)$ is increased, because blood viscosity is proportional to $\alpha_{\text {Blood }}$ In addition, the enhanced blood viscosity contributes to increasing fluidic resistance in the microfluidic channel. Therefore, the velocity of blood flow $\left(\langle U\rangle_{\mathrm{DAC}},\langle U\rangle_{\mathrm{BRG}}\right)$ and the supplied blood volume $\left(\Delta V_{\mathrm{DAC}}, \Delta V_{\mathrm{BRG}}\right)$ are decreased with increasing concentration of Dextran solution. Since shear rate is estimated to be $4.1 \times$ $10^{5} \mathrm{~s}^{-1}$, blood flow behaves as Newtonian fluid. At a high shear rate, blood flow is strongly influenced by deformability of RBCs. Thus, the enhanced aggregation of RBCs does not change blood flow in the microfluidic channel noticeably.

On the other hand, microscopic images of Figure 4C show dynamic behaviors of RBC aggregation, depending on blood flow condition in the DAC [(a) before closing the outlet (A), (b) short-time elapsed after closing the outlet (A), and (c) long-time elapsed after closing the outlet (A)]. After closing the outlet (A), blood flow in the DAC is stopped immediately. Thereafter, RBCs tends to be aggregated or clustered in the upper region of the DAC. Blood sample added with concentration of Dextran solution $\left(C_{\mathrm{GA}}=10 \mathrm{mg} / \mathrm{mL}\right)$ exhibits higher RBC aggregation, compared with normal RBCs in PBS suspension (i.e., control blood sample). To quantify dynamic behavior of RBC aggregation in the upper region of DAC, the average value of image intensity $(<I>)$ in a specific ROI $(475 \times$ 50 pixels) was estimated using commercial software (Matlab, Mathworks, U.S.A.). As a result, the enhanced RBC aggregation induces different dynamic behaviors, compared with the control blood sample. In other words, the image intensity $(\langle I\rangle)$ of control blood sample is gradually increased with increasing elapse of time. However, $\langle I>$ of the blood sample mixed with Dextran solution is saturated in a short time. According to previous study, ${ }^{6}$ RBC aggregation was quantified by obtaining a time constant from temporal variations of the image intensity. Taking into account the fact that blood sample mixed with Dextran solution has much smaller time constant than the control blood sample, Dextran solution contributes to increasing $\mathrm{RBC}$ aggregation positively.

These experimental results indicate that blood sample with highly aggregated RBCs has little influence on change of blood flow due to cytoadherence in the bridge channel. In addition, the proposed method has a strong potential for evaluating RBC aggregation of blood sample.

Detection of Pf-Infected RBCs. As a clinical trial, the proposed method was directly applied to detect blood samples with Pf-infected RBCs. The average velocities of blood flow $\left(\langle U\rangle_{\mathrm{BRG}},\langle U\rangle_{\mathrm{DAC}}\right)$, the delivered blood volumes $\left(\Delta V_{\mathrm{DAC}}\right.$, $\left.\Delta V_{\mathrm{BRG}}\right)$, and the blood-filled width $\left(\alpha_{\text {Blood }}\right)$ are quantitatively evaluated with increasing parasitemia and progressing malariainfection stage from the ring to the schizont. In this clinical demonstration, the flow rates of the Pf-infected blood sample and reference fluid were supplied into the microfluidic device $\left(L_{\text {DAC }}=250 \mu \mathrm{m}\right)$ at the same flow rate of $Q_{\text {Blood }}=Q_{\text {Ref }}=1 \mathrm{~mL} /$ h. The hematocrit of the Pf-infected blood was adjusted to 50\% by adding Pf-infected RBCs into $1 \times$ PBS. Each experiment was repeated three times $(n=3)$. 
A

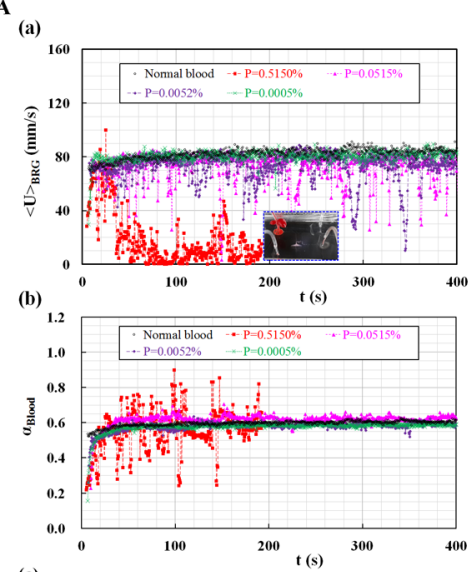

B (a)

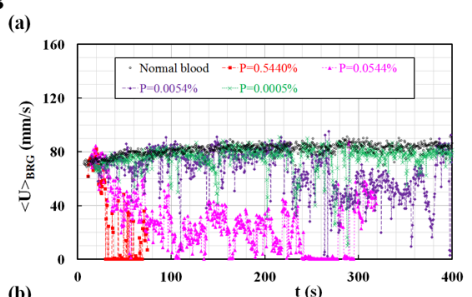

(b)

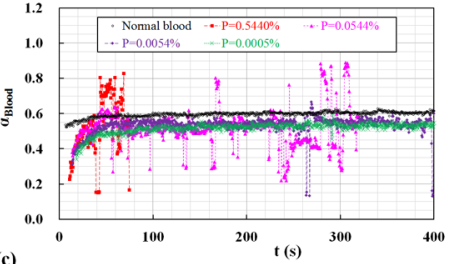

C

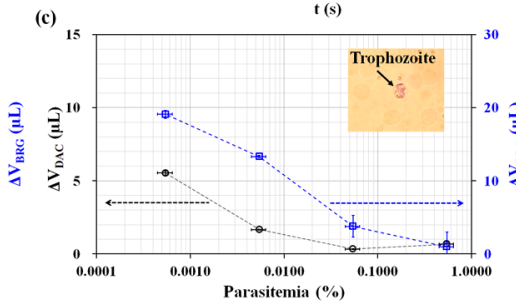

(a)

(b)
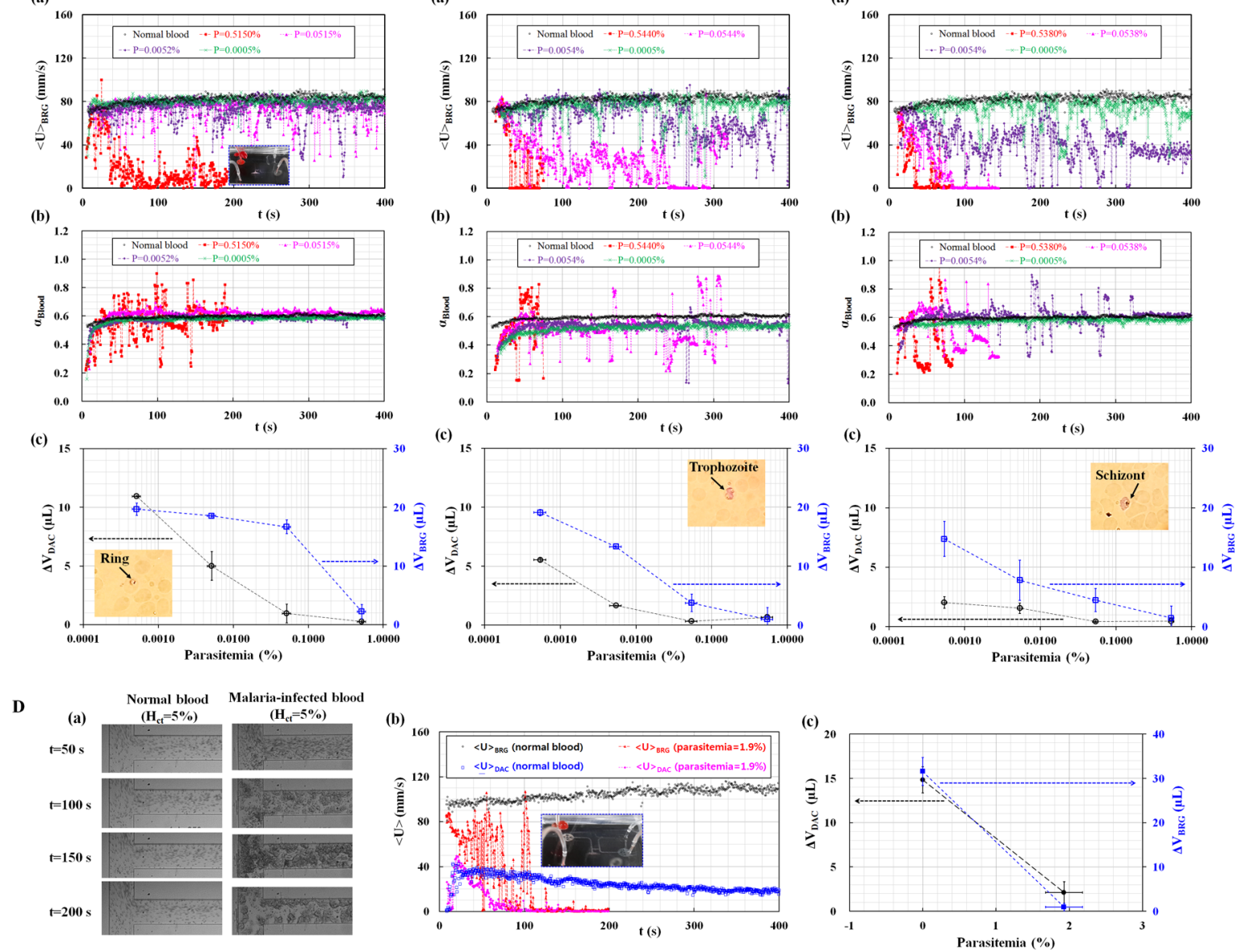

Figure 5. Clinical demonstration of the proposed method for the detection of Pf-infected RBCs. The delivered blood volume $\left(\Delta V_{\mathrm{DAC}}, \Delta V_{\mathrm{BRG}}\right)$, blood-filled width $\left(\alpha_{\text {Blood }}\right)$, and blood velocity $\left(\langle U\rangle_{\text {BRG }}\right)$ were quantitatively evaluated, with increasing parasitemia and progressing malaria-infection stage from the ring to the schizont. Here, the hematocrit of the blood sample with Pf-infected RBCs was fixed at 50\%. (A) Detection of ring-stage Pfinfected RBCs. (a) Temporal variations of $\langle U\rangle_{\text {BRG }}$ with respect to parasitemia $(P)[(a) P=0.0005 \pm 0.0001 \%$, (b) $P=0.0052 \pm 0.0006 \%$, (c) $P=$ $0.0515 \pm 0.0064 \%$, and (d) $P=0.515 \pm 0.0635 \%]$. (b) Temporal variations of $\alpha_{\text {Blood }}$ with respect to parasitemia. (c) Temporal variations of $\Delta V_{\text {DAC }}$ and $\Delta V_{\text {BRG }}$ with respect to parasitemia. (B) Detection of trophozoite-stage Pf-infected RBCs. (a) Temporal variations of $\langle U\rangle_{\text {BRG }}$ with respect to parasitemia $(P)[(\mathrm{a}) P=0.0005 \pm 0.0001 \%$, (b) $P=0.0054 \pm 0.001 \%$, (c) $P=0.0544 \pm 0.0095 \%$, and (d) $P=0.544 \pm 0.095 \%]$. (b) Temporal variations of $\alpha_{\text {Blood }}$ with respect to parasitemia. (c) Temporal variations of $\Delta V_{\mathrm{DAC}}$ and $\Delta V_{\mathrm{BRG}}$ with respect to parasitemia. (C) Detection of schizontstage Pf-infected RBCs. (a) Temporal variations of $\langle U\rangle_{\text {BRG }}$ with respect to parasitemia $(P)[($ a $) P=0.0005 \pm 0.0001 \%$, (b) $P=0.0054 \pm 0.0005 \%$, (c) $P=0.0538 \pm 0.0047 \%$, and (d) $P=0.538 \pm 0.0466 \%$ ]. (b) Temporal variations of $\alpha_{\text {Blood }}$ with respect to parasitemia. (c) Temporal variations of $\Delta V_{\mathrm{DAC}}$ and $\Delta V_{\mathrm{BRG}}$ with respect to parasitemia. (D) At a fixed hematocrit of $H_{\mathrm{ct}}=5 \%,\langle U\rangle_{\mathrm{BRG}},\langle U\rangle_{\mathrm{DAC}}, \Delta V_{\mathrm{DAC}}$, and $\Delta V_{\mathrm{BRG}}$ for quantitatively evaluated values of the normal and Pf-infected blood samples were compared. (a) Sequential microscopic images for the normal and Pf-infected blood samples with respect to elapse of time. (b) Temporal variations of $\langle U\rangle_{\text {BRG }}$ and $\langle U\rangle_{\text {DAC }}$ with respect to parasitemia $(P)[(a) P=0$, and (b) $P$ $=1.9 \%]$. (c) Variations of $\Delta V_{\mathrm{DAC}}$ and $\Delta V_{\mathrm{BRG}}$ with respect to parasitemia.

First, using blood samples composed of ring-stage Pf-infected RBCs, the average velocities in the DAC and bridge channel $\left(\langle U\rangle_{\text {DAC }},\langle U\rangle_{\text {BRG }}\right)$ were evaluated using a time-resolved microPIV technique. Figure 5A-(a) shows temporal variations of $\langle U\rangle_{\text {BRG }}$ with respect to parasitemia $(P)[($ a $) P=0.0005 \pm$ $0.0001 \%$, (b) $P=0.0052 \pm 0.0006 \%$, (c) $P=0.0515 \pm$ $0.0064 \%$, and (d) $P=0.515 \pm 0.0635 \%]$. For a blood sample composed of Pf-infected RBCs with parasitemia of $0.515 \%$, $\langle U\rangle_{\mathrm{BRG}}$ exhibits distinctly unstable variations. In other words, after a certain period of time, the blood flow in the bridge channel stops and runs repeatedly. Due to continuous supply of blood flow by a syringe pump, hydrostatic pressure is gradually increased in the microfluidic channel. Thus, blood leakage occurs at the inlet (A) as shown in inset of Figure 5A-(a). Although the $\langle U\rangle_{\text {BRG }}$ of the blood sample composed of RBCs with parasitemia ranging from $0.0052 \%$ to $0.0515 \%$ shows slightly unstable flow behavior, blood leakage does not occur at the inlet $(\mathrm{A})$ during delivery of blood flow. In addition, the blood sample composed of Pf-infected RBCs with parasitemia less than $0.0004 \%$ exhibits stable fluidic motions. Figure 5A-(b) shows temporal variations of $\alpha_{\text {Blood }}$ with respect to parasitemia. The $\alpha_{\text {Blood }}$ of the blood sample composed of Pf-infected RBCs with parasitemia of $0.515 \%$ has unstable fluctuations with elapse of time. To quantify the deformability of blood sample composed of Pf-infected RBCs, variations of $\Delta V_{\mathrm{DAC}}$ were examined with varying parasitemia. As shown in Figure 5A-(c), $\Delta V_{\text {DAC }}$ is largely decreased with increasing parasitemia. Because it is difficult for Pf-infected RBCs to pass through the microfluidic filters, $\Delta V_{\mathrm{DAC}}$ tends to decrease with increasing parasitemia. Inset of Figure 5A-(c) shows microscopic images of ring-stage Pf-infected RBCs. In addition, the $\Delta V_{\mathrm{BRG}}$ of Pfinfected RBCs with parasitemia of $0.515 \%$ is significantly 
decreased due to presence of unstable blood flows in the microfluidic channel. This experimental result supports that the proposed method is capable of detecting ring-stage Pf-infected RBCs with high parasitemia larger than $0.515 \%$, by monitoring flow velocity of blood sample, delivered blood volume, bloodfilled width, and port leakage.

Second, using blood samples composed of trophozoite-stage Pf-infected RBCs, the average velocities in the DAC and bridge channel were evaluated. Figure 5B-(a) shows temporal variations of $\langle U\rangle_{\mathrm{BRG}}$ with respect to parasitemia $(P)[(\mathrm{a}) P$ $=0.0005 \pm 0.0001 \%$, (b) $P=0.0054 \pm 0.001 \%$, (c) $P=0.0544$ $\pm 0.0095 \%$, and (d) $P=0.544 \pm 0.095 \%]$. As shown in Figures 5B-(a) and 5B-(b), for a blood sample composed of Pf-infected RBCs with parasitemia larger than $0.0544 \%,\langle U\rangle_{\text {BRG }}$ and $\alpha_{\text {Blood }}$ exhibit significantly unstable variations. In addition, blood leakage occurs at the inlet (A) as shown in Figure S1 (Supporting Information). As depicted in Figure 5B-(c), the delivered blood volumes $\left(\Delta V_{\mathrm{DAC}}\right.$ and $\left.\Delta V_{\mathrm{BRG}}\right)$ are largely decreased with increasing parasitemia. This experimental result indicates that the proposed method can detect trophozoitestage Pf-infected RBCs with parasitemia larger than $0.0544 \%$ by monitoring various parameters handled in this study.

Third, using blood sample composed of schizont-stage Pfinfected RBCs, the average velocities in the DAC and bridge channel were evaluated. Figure 5C-(a) shows temporal variations of $\langle U\rangle_{\mathrm{BRG}}$ with respect to parasitemia $(P)[(\mathrm{a}) P$ $=0.0005 \pm 0.0001 \%$, (b) $P=0.0054 \pm 0.0005 \%$, (c) $P=$ $0.0538 \pm 0.0047 \%$, and (d) $P=0.538 \pm 0.0466 \%]$. As shown in Figures 5C-(a) and 5C-(b), for a blood sample composed of Pfinfected RBCs with parasitemia larger than $0.0054 \%,\langle U\rangle_{\text {BRG }}$ and $\alpha_{\text {Blood }}$ exhibit significantly unstable variations. In addition, blood leakage occurs at the inlet (A) as shown in Figure S1 (Supporting Information). As depicted in Figure 5B-(c), $\Delta V_{\mathrm{DAC}}$ and $\Delta V_{\mathrm{BRG}}$ are significantly decreased with increasing parasitemia. This experimental result supports that the proposed method has ability to detect schizont-stage Pfinfected RBCs with parasitemia larger than $0.0054 \%$.

However, the reason for unstable fluidic behaviors of malariainfected blood samples in the microfluidic channel remains unknown. According to a previous study, ${ }^{14}$ the cytoadherence of Pf-infected RBCs to the surface caused unstable fluidic behavior. Thus, to observe the cytoadherence of Pf-infected RBCs specifically in the microfluidic channel, the hematocrit of malaria-infected blood sample (parasitemia $=1.9 \%$ ) largely decreased to 5\%. As shown in Figure 5D-(a), microscopic images were sequentially captured and compared for the normal and malaria-infected blood samples. Pf-infected RBCs exhibited remarkable cytoadherence to the microfluidic channel, which hindered blood flow in the microfluidic channels compared with normal blood sample. Using a timeresolved micro-PIV technique, the temporal variations in $\langle U\rangle_{\text {BRG }}$ and $\langle U\rangle_{\text {DAC }}$ were measured for the normal and malaria-infected blood samples. As shown in Figure 5D-(b), $\langle U\rangle_{\text {BRG }}$ of the malaria-infected blood samples showed unstable variations compared with normal blood samples. $\langle U\rangle_{\text {DAC }}$ of the malaria-infected blood sample became zero within approximately $60 \mathrm{~s}$. As shown in the inset of Figure 5D-(b), blood leakage occurred at the inlet (A). However, $\langle U\rangle_{\text {DAC }}$ of the normal blood sample continuously decreased in the DAC channel for $400 \mathrm{~s}$. Using the temporal variations in $\langle U\rangle_{\text {DAC }}$ and eq 4 , the blood volumes delivered into the DAC $\left(\Delta V_{\text {DAC }}\right)$ for normal and malaria-infected blood samples were compared. As shown in Figure $5 \mathrm{D}-(\mathrm{c}), \Delta V_{\mathrm{DAC}}$ of the malaria-infected blood sample was much lower than that of the normal blood sample. In addition, $\Delta V_{\mathrm{BRG}}$ of the malaria-infected blood sample is much smaller than that of the normal blood sample, because Pfinfected RBCs hinders blood flow in the microfluidic channels, compared with normal RBCs.

On the basis of these demonstrations, the proposed method could be used to detect Pf-infected RBCs effectively by monitoring average velocity of blood flow $\left(\langle U\rangle_{\text {BRG }},\langle U\rangle_{\text {DAC }}\right)$, delivered blood volume $\left(\Delta V_{\mathrm{DAC}}, \Delta V_{\mathrm{BRG}}\right)$, blood-filled width $\left(\alpha_{\text {Blood }}\right)$, and port leakage with good accuracy. However, this proposed method has a practical limitation on POCT (PointOf-Care-Test) or clinical setting. In other words, the microfluidic device can be only used in a laboratory environment equipped with a microscope. Thus, in resourcescare clinical environments, the microscope should be replaced by an analytical sensor which can detect temporal variations of blood flows in the microfluidic device. As a future research work, various analytical techniques, including pressure sensor, flow-rate sensor, vibrational spectroscopy, ${ }^{18}$ and electrodebased impedance analyzer, will be considered to effectively detect malaria-infected RBCs.

\section{CONCLUSION}

Simultaneous quantification of biophysical properties of blood samples was proposed to detect Pf-infected RBCs by measuring the velocity fields of blood flow in a microfluidic device. The microfluidic device was mimicked from a Wheatstone-bridge electric circuit. To measure RBC deformability effectively, the left lower side channel was designed to have a DAC with parallel microfluidic filters $(N=43$, gap $=2 \mu \mathrm{m}$, and overall length $=400 \mu \mathrm{m}$ ). After supplying blood sample and $1 \times \mathrm{PBS}$ solution at the same flow rate of $Q_{\text {Blood }}=Q_{\mathrm{PBS}}=1 \mathrm{~mL} / \mathrm{h}$, the hemodynamic variations in the microfluidic channels were quantified using a time-resolved micro-PIV technique. Based on experimental investigations, blood flow in the DAC stopped because of successive clogging of RBCs in the microfluidic filters, especially within $200 \mathrm{~s}$. Thus, the blood volume delivered into the DAC $\left(\Delta V_{\mathrm{DAC}}\right)$ for $200 \mathrm{~s}$ was suggested as a deformability index. Thereafter, using parallel flows in a single channel of the microfluidic device, the viscosity ratio of blood sample to the reference fluid was quantified by monitoring blood-filled width $\left(\alpha_{\text {Blood }}\right)$. Furthermore, the blood volume delivered into the bridge channel $\left(\Delta V_{\mathrm{BRG}}\right)$ for $100 \mathrm{~s}$ was suggested as a cytoadherence index. Thus, the deformability, viscosity, and cytoadherence of blood samples that were determined from hemodynamic variations in the microfluidic channel were used to detect Pf-infected RBCs in a label-free and high-throughput manner.

The effects of four factors, namely, the length of microfluidic filter $\left(L_{\text {DAC }}\right)$, blood flow rate $\left(Q_{\text {Blood }}\right)$, hematocrit $\left(H_{\mathrm{ct}}\right)$, and base solution, were experimentally evaluated. On the basis of the results, the hematocrit of blood sample was fixed at 50\%, and the length of the microfluidic filter in the DAC was set at $250 \mu \mathrm{m}$.

Homogeneous hardened blood sample and heterogeneous blood samples partially mixed with normal and hardened RBCs were prepared to evaluate variations in RBC deformability and viscosity caused by small variations in subpopulations of RBCs. The blood volume supplied into the DAC $\left(\Delta V_{\text {DAC }}\right)$ was more effective than the blood-filled width $\left(\alpha_{\text {Blood }}\right)$ in detecting heterogeneous blood samples with hardened blood volume fraction $(\psi)(\psi<5 \%)$. 
Blood sample composed of RBCs with enhanced aggregation was evaluated by employing the proposed method. RBC aggregation was gradually enhanced by adding normal RBCs into Dextran solution. Blood sample with highly aggregated RBCs has little influence on the change of blood flow in the bridge channel. Furthermore, RBC aggregation of blood sample was evaluated by monitoring temporal variations of image intensity $(<I>)$ in the DAC, after closing the outlet (A).

As a clinical demonstration, the proposed method was applied to detect blood samples composed of Pf-infected RBCs. When parasitemia was greater than $0.515 \%$ for ring stage, $0.0544 \%$ for trophozoite stage, and $0.0054 \%$ for schizont stage, average velocity of blood flow and blood-filled width showed unstable fluctuations. After a certain period, the blood flow stopped and ran repeatedly. Finally, the blood leakage occurred in the inlet (A). This unstable fluidic behavior was checked by monitoring the cytoadherence of Pf-infected RBCs to the surface of the microfluidic channel. $\Delta V_{\mathrm{DAC}}$ as a deformability index and $\Delta V_{\mathrm{BRG}}$ as cytoadherence index significantly decreased with increasing parasitemia.

These results indicated that the proposed method could be used to detect Pf-infected RBCs by monitoring average velocities of blood flow $\left(\langle U\rangle_{\mathrm{BRG}},\langle U\rangle_{\mathrm{DAC}}\right)$, delivered blood volumes $\left(\Delta V_{\mathrm{DAC}}, \Delta V_{\mathrm{BRG}}\right)$, blood-filled width $\left(\alpha_{\mathrm{Blood}}\right)$, and port leakage with good accuracy. Furthermore, the experimental procedures for a blood sample of $0.2 \mathrm{~mL}$ with a high hematocrit level of $50 \%$ were completed within a short time period $(\sim 5$ $\mathrm{min})$. The proposed method will be tested further to evaluate the blood biophysical properties of blood samples related to various hematological diseases.

\section{ASSOCIATED CONTENT}

\section{S Supporting Information}

The Supporting Information is available free of charge on the ACS Publications website at DOI: 10.1021/acs.analchem.5b04874.

Figure of supplemental data showing blood leakages in the inlet with increasing parasitemia and progressing malaria-infection stage from the ring to the schizont (PDF)

\section{AUTHOR INFORMATION}

\section{Corresponding Author}

*E-mail: sjlee@postech.ac.kr. Fax: +82-54-279-3199.

\section{Notes}

The authors declare no competing financial interest.

\section{ACKNOWLEDGMENTS}

This work was partially supported by the National Research Foundation (NRF) of Korea grant funded by the Korean Government (MSIP) (No. 2008-0061991), the research fund from Chosun University in 2014, and the Basic Science Research Program through NRF funded by the Ministry of Science, ICT, and Future Planning (NRF2015R1C1A1A02036624).

\section{REFERENCES}

(1) Di Carlo, D. JALA 2012, 17, 32-42.

(2) Zheng, Y.; Nguyen, J.; Wei, Y.; Sun, Y. Lab Chip 2013, 13, 24642483.

(3) Fedosov, D. A.; Caswell, B.; Suresh, S.; Karniadakis, G. E. Proc. Natl. Acad. Sci. U. S. A. 2011, 108, 35-39.
(4) Deplaine, G.; Safeukui, I.; Jeddi, F.; Lacoste, F.; Brousse, V.; Perrot, S.; Biligui, S.; Guillotte, M.; Guitton, C.; Dokmak, S.; Aussilhou, B.; Sauvanet, A.; Hatem, D. C.; Paye, F.; Thellier, M.; Mazier, D.; Milon, G. v.; Mohandas, N.; Mercereau-Puijalon, O.; David, P. H.; Buffet, P. A. Blood 2011, 117, e88-e95.

(5) Peng, W. K.; Kong, T. F.; Ng, C. S.; Chen, L.; Huang, Y.; Bhagat, A. A. S.; Nguyen, N.-T.; Preiser, P. R.; Han, J. Nat. Med. 2014, 20, 1069-1073.

(6) Kang, Y. J.; Ha, Y.-R.; Lee, S.-J. Biomicrofluidics 2014, 8, 044114.

(7) Herricks, T.; Antia, M.; Rathod, P. K. Cell. Microbiol. 2009, 11, 1340-1353.

(8) Rosenbluth, M. J.; Lam, W. A.; Fletcher, D. A. Lab Chip 2008, 8, 1062-1070.

(9) Shelby, J. P.; White, J.; Ganesan, K.; Rathod, P. K.; Chiu, D. T. Proc. Natl. Acad. Sci. U. S. A. 2003, 100, 14618-14522.

(10) Bow, H.; Pivkin, I. V.; Diez-Silva, M.; Goldfless, S. J.; Dao, M.; Niles, J. C.; Suresh, S.; Han, J. Lab Chip 2011, 11, 1065-1073.

(11) Hou, H. W.; Bhagat, A. A. S.; Chong, A. G. L.; Mao, P.; Tan, K. S. W.; Han, J.; Lim, C. T. Lab Chip 2010, 10, 2605-2613.

(12) Guo, Q.; Reiling, S. J.; Rohrbach, P.; Ma, H. Lab Chip 2012, 12, $1143-1150$

(13) Du, E.; Ha, S.; Diez-Silva, M.; Dao, M.; Suresh, S.; Chandrakasan, A. P. Lab Chip 2013, 13, 3903-3909.

(14) Antia, M.; Herricks, T.; Rathod, P. K. PLoS Pathog. 2007, 3, e99.

(15) Park, Y.; Diez-Silva, M.; Popescu, G.; Lykotrafitis, G.; Choi, W.; Feld, M. S.; Suresh. Proc. Natl. Acad. Sci. U. S. A. 2008, 105, 1373013735.

(16) Mauritz, J. M. A.; Tiffert, T.; Seear, R.; Lautenschläger, F.; Esposito, A.; Lew, V. L.; Guck, J.; Kaminski, C. F. J. Biomed. Opt. 2010, 15, 030517.

(17) Nam, J.; Huang, H.; Lim, H.; Lim, C.; Shin, S. Anal. Chem. 2013, 85, 7316-7323.

(18) Mitchell, A. L.; Gajjar, K. B.; Theophilou, G.; Martin, F. L.; Martin-Hirsch, P. L. J. Biophotonics 2014, 7, 153-165. 